\title{
EchoGéo
}

ECHOGEO- $\quad 41 \mid 2017$

Un état des lieux du Brésil en 2017

\section{Brésil : un écho géographique sur l'émergence}

Jean-Louis Chaléard

\section{(2) OpenEdition}

Journals

Electronic version

URL: https://journals.openedition.org/echogeo/15101

DOI: 10.4000/echogeo.15101

ISSN: 1963-1197

\section{Publisher}

Pôle de recherche pour l'organisation et la diffusion de l'information géographique (CNRS UMR 8586)

Electronic reference

Jean-Louis Chaléard, "Brésil : un écho géographique sur l'émergence", EchoGéo [Online], 41 | 2017,

Online since 28 September 2017, connection on 01 August 2021. URL: http://journals.openedition.org/ echogeo/15101; DOl: https://doi.org/10.4000/echogeo.15101

This text was automatically generated on 1 August 2021

EchoGéo est mis à disposition selon les termes de la licence Creative Commons Attribution - Pas d'Utilisation Commerciale - Pas de Modification 4.0 International (CC BY-NC-ND) 


\title{
Brésil : un écho géographique sur l'émergence
}

\author{
Jean-Louis Chaléard
}

1 Le présent numéro d'ÉchoGéo s'attache à un grand pays émergent, situé sur le souscontinent latino-américain, le Brésil. Celui-ci a souvent fait la une de l'actualité ces dernières années, par l'organisation de grands évènements (Coupe du monde de football en 2014, Jeux olympiques en 2016), par son dynamisme économique, et par des aspects plus troubles ou critiques: corruption à l'intérieur de l'État et des grands groupes économiques, mise en accusation de l'ancien président Luiz Inácio « Lula » da Silva, destitution de la présidente Dilma Rousseff, violences urbaines, etc. Un dossier sur le Brésil fait forcément écho à celui que nous avions proposé en 2015 sur l'Inde, autre grand pays émergent, situé sur un autre continent. H. Théry y fait d'ailleurs allusion dans l'introduction du dossier Sur le Champ. À lire les introductions de ces deux dossiers et les articles qui suivent comment ne pas voir les mêmes questions, qui se posent à des grands pays émergents: poids de la métropolisation, violence des contrastes sociaux et spatiaux, économie à plusieurs vitesses... Mais bien sûr la comparaison atteint vite ses limites. Les deux géants diffèrent par leurs densités de population, l'histoire de leur peuplement, leur organisation spatiale. L'Inde est un monde plein, où des États se sont structurés depuis longtemps, où se sont épanouies d'anciennes civilisations. Le Brésil est un pays encore pionnier, peuplé largement par des migrants, volontaires (européens) ou non (esclaves africains), sans compter que même les histoires coloniales, britannique pour l'un, portugaise pour l'autre, diffèrent fortement.

2 Le dossier Sur le Champ, «Un état des lieux du Brésil en 2017 », permet d'appréhender ces questions et de présenter les grands thèmes d'une géographie actuelle du Brésil. Dans son introduction, H. Théry fournit des éléments généraux pour comprendre la situation du pays et souligne les contrastes du peuplement, en particulier entre des régions littorales densément peuplées et un intérieur beaucoup plus faiblement occupé. Les articles qui suivent approfondissent différents aspects, mettant en cause plusieurs fois des idées reçues (sur la métropolisation ou l'Amazonie, par exemple). Trois textes 
s'intéressent aux contrastes spatiaux. Parmi eux, deux se focalisent sur les villes. La croissance urbaine se concentre-t-elle dans les métropoles? C. Chatel et al. montrent que la situation est plus complexe et que les deux principales métropoles, Sao Paulo et Rio de Janeiro, ont une croissance inférieure à la moyenne nationale. À une échelle plus fine, en s'appuyant sur le cas de Vila Autódromo et les travaux d'aménagement pour les Jeux olympiques qui y ont eu lieu, C. Zanotelli met en évidence la perpétuation de processus d'exclusion des classes populaires de la propriété foncière et immobilière urbaine. Qu'en est-il des espaces ruraux ? À propos de leurs contrastes internes, C. A Boechat et al. s'intéressent à la genèse de la question agraire au Brésil, engendrée par des processus d'expropriation reposant sur l'esclavage ou l'appropriation privée de la terre.

Plusieurs articles se concentrent sur des questions qui ont fait et font encore débat au Brésil : l'environnement, les Indiens, et bien sûr l'Amazonie où ces deux questions se rencontrent. N.A. de Mello-Théry mène une réflexion sur les politiques environnementales du pays, mettant en évidence les avancées et les reculs sous les gouvernements de «Lula » et de Dilma Rousseff et les incertitudes actuelles. À partir de l'utilisation de la classification des climats de Köppen, V. Dubreuil et al. proposent une géographie fine et nuancée des différents types de climats annuels, des plus tropicaux aux tempérés. Autre question brûlante: les Indiens. F. M. Le Tourneau fait un panorama historique de la manière dont la question amérindienne a été considérée au Brésil depuis la colonisation portugaise, puis analyse la consolidation d'un patrimoine foncier amérindien avec la Constitution de 1988 et les inflexions dans un sens défavorable actuelles. Ces questions se retrouvent bien sûr en Amazonie, objet de trois articles. C. Broggio et M. Droulers s'intéressent aux équipements hydro-électriques, aspect du front pionnier. À partir de l'analyse du cas du barrage trentenaire de Tucurui (Pará), elles mettent en évidence localement des processus de résilience caractéristiques d'une phase post-pionnière et en tirent des éléments de réflexion qui peuvent être apportés à propos de barrages plus récents. F. Laurent et al. montrent que l'Amazonie a amorcé depuis quelques années une baisse de la déforestation couplée à un développement économique, mais que le processus est inégal, les petites exploitations familiales étant à l'écart du mouvement mené par les exploitations grandes et moyennes qui intensifient et diversifient leurs systèmes de production. T. Schor et G.S. Azenha, à partir de l'examen des régimes alimentaires et de l'acquisition des aliments, mènent une étude critique du développement durable en Amazonie.

4 Les deux derniers articles traitent des questions de politique et d'aménagement régional au Brésil. S. Affonso da Silva part d'une analyse des différentes interprétations des inégalités régionales de quatre intellectuels brésiliens puis des politiques principales mises en place par le gouvernement fédéral depuis le milieu du $\mathrm{XX}^{\mathrm{e}}$ siècle pour essayer de comprendre le rôle des inégalités régionales dans les questions de développement au Brésil. Le dernier texte de R. C. Gadioli dos Santos et al. étudie la place de Brasilia dans la politique nationale pour le développement régional (PNDR), soulignant que sa gouvernance fait l'objet de conflits et d'inégalités spatiales fortes entre le centre et les périphéries.

5 Dans le dossier alternent des textes généraux, abordant les questions à l'échelle du pays (sur les villes, sur les espaces ruraux, sur l'environnement, sur les Amérindiens...), et des textes qui se focalisent sur des espaces plus réduits (sur un quartier de Rio, sur des 
barrages en Amazonie, sur Brasilia...). Les premiers permettent d'avoir une réflexion générale sur le pays et sur les processus majeurs de son évolution. Les seconds fournissent des analyses géographiquement plus précises, qui autorisent l'approfondissement de points majeurs. De nombreux textes s'appuient sur l'histoire pour mettre en évidence les singularités actuelles des questions étudiées, pour voir comment la situation présente s'inscrit dans un mouvement ancien ou pour examiner les évolutions sur un pas de temps plus ou moins long. C'est vrai pour C. Arruda Boechat et al. qui proposent une véritable archéologie de la question agraire au Brésil. C'est vrai plus encore pour F.-M. Le Tourneau qui inscrit la dépossession territoriale des Indiens dans l'histoire coloniale et post-coloniale du Brésil. S. Affonso da Silva reprend les évolutions des politiques du gouvernement brésilien depuis le milieu du $\mathrm{XX}^{\mathrm{e}}$ siècle pour montrer qu'elles ont été marquées par des idéologies et des projets politiques sous-jacents. La durée est aussi au cœur du texte de N. A. de Mello-Théry qui étudie l'évolution des politiques environnementales sous Lula et Dilma Rousseff, et de celui de C. Broggio et M. Droulers sur les barrages et les usines hydro-électriques d'Amazonie, même si les pas de temps sont plus courts.

Plusieurs articles insistent sur des questions de méthode ou l'analyse de concepts qui vont bien au-delà du cas du Brésil et peuvent servir à une réflexion générale. Notamment, C. Chatel et al. s'interrogent sur la définition de l'urbain et les conséquences du choix des définitions retenues. L'article de V.Dubreuil et al. est largement méthodologique, montrant l'intérêt de la classification de Köbben à condition d'en dépasser les limites (car fondée sur des valeurs moyennes). T. Schor et G. S. Azenha proposent aussi une méthode originale, fondée sur une écologie politique des régimes alimentaires, pour éclairer les contradictions actuelles du développement. Plusieurs textes s'appuient sur une réflexion conceptuelle préalable, comme celui de C. Zanotelli qui revient sur les thèmes du droit à la ville et de la justice spatiale à partir des travaux de H. Lefebvre et de B. Bret. R. C. Gadioli dos Santos et al. appuient leur analyse sur un corpus théorique et méthodologique (P. Boudieu, N. Fligstein...) pour analyser le modèle de gouvernance à Brasilia.

7 La rubrique Sur l'Image complète l'étude sur le Brésil avec une série de photographies commentées de H. Théry, sur Sao Paulo, principale métropole brésilienne. Les images rendent plus concrets le modernisme et la puissance de la ville, le luxe de certains quartiers, la misère d'autres. Sao Paulo symbole des contrastes d'un grand pays émergent?

Dans un tout autre registre, la rubrique Sur l'Écrit donne la parole à une écrivaine de renom, Marie-Hélène Lafon, dont les textes, qu'ils soient romans, nouvelles ou miniatures, ont une manière étrangement géographique de dire "les pays", ces campagnes reculées du Massif Central où la rudesse du quotidien, la ruralité brute, entre nature, travail agricole et âpreté des vies, semblent appartenir au passé et sont confrontées à la modernité. Dans la façon qu'elle a de dire son rapport «ombilical » au paysage et à l'enracinement agricole, elle livre de saisissants témoignages des marges rurales et de l'appartenance aux lieux et aux paysages, de l'intérieur.

Dans la rubrique Sur le Métier S. Gueben-Vernière s'interroge sur les représentations mentales, recueillies en général de manière individuelle. Elle propose d'envisager des ateliers collaboratifs pour l'étude des représentations spatiales émanant de plusieurs groupes d'acteurs qui doivent se coordonner. Appliqué au cas d'une crise liée à une crue majeure de la Seine en Île de France, l'article met en évidence l'intérêt 
opérationnel d'une telle démarche. La conclusion propose une synthèse des techniques utilisées pour recueillir des représentations spatiales en comparant avec une autre crise analysée dans un précédent numéro d'Echogéo. L'écho pour élargir et approfondir la connaissance. 